\title{
La adicción a los videojuegos. Una revisión
}

\author{
Tejeiro, $\mathbf{R}$.
}

Universidad Nacional de Educación a Distancia.

Enviar correspondencia a:

Ricardo Tejeiro Salguero. Universidad Nacional de Educación a Distancia. Los Jazmines, 46, 11207 Algeciras. Telf. 956570841. tejeirosalguero@terra.es

\section{RESUMEN}

A distintos niveles parece darse por cierta la existencia de la adicción a los videojuegos. Este trabajo revisa de manera crítica las investigaciones sobre este tema. Entre las principales conclusiones se incluyen la falta de estudios centrados específicamente en la adicción a los videojuegos y las notables carencias de muchas de las investigaciones. Ningún estudio hace explícito el modelo de adicción del que parte, y los conceptos utilizados no suelen ser explicados adecuadamente. La mayoría de los trabajos se basan en encuestas generales de dudosa validez y fiabilidad, mientras que otros se centran únicamente en determinados tipos de máquinas, con lo que sus resultados son escasamente generalizables. No se dispone de un sistema diagnóstico válido y fiable para esa presunta adicción, y las adaptaciones propuestas no son aplicables a todos los sistemas de juego o se desconocen sus propiedades psicométricas. La mayoría de los trabajos revisados tienden a señalar que, para algunos sujetos, el uso de los videojuegos constituye un problema. Sin embargo, los conocimientos actuales no permiten determinar la naturaleza y el origen de dicho problema. Se finaliza comentado posibles líneas de trabajo futuro.

\begin{abstract}
Video game addiction seems to be a widely accepted disorder. This work reviews in a critical manner the literature and the state of the art about this topic. The scarcity of works focused specifically on video game addiction, as well as the weakness of many of the investigations are commented as main conclusions. None of the studies makes explicit the model of addiction that constitutes its reference, and concepts are only seldom appropriately explained. Most of the works are based on general surveys of doubtful validity and reliability, while others are focused only on certain types of video machines, thus limiting the generalisation of their results. No valid and reliable diagnostic system is so far available to measure that presumed addiction, and the adaptations that have been proposed are not applicable to all video game systems or their psychometric properties are unknown. Certainly, a majority of works report that, for some subjects, video game use constitutes a problem. Nevertheless, the nature and the possible origins of this problem remain as unsolved questions. Finally, possible lines for future work are reviewed.
\end{abstract}

Key words: addiction, dependency, video games, review.

\section{INTRODUCCIÓN}

$\mathbf{U}$ no de los efectos negativos que han sido asociados con más frecuencia al uso de videojuegos ha sido su potencial para crear adicción. Con frecuencia, la polémica se ha desarrollado a base de experiencias personales, observaciones anecdóticas, y objeciones de tipo moralista. Esta discusión ha traspasado el ámbito científico para convertirse en un tema de preocupación popular, fomentada por artículos poco rigurosos en los medios de comunicación. Lamentablemente, hay organismos oficiales que se han lanzado a la arena de la polémica sosteniendo posturas alarmistas que no sólo no gozan del respaldo de las investigaciones sino que, en algunos casos, se oponen a las mismas. Un ejemplo de ello lo tenemos en el texto que, con el título de "Juegos peligrosos", difunde el Servicio de Juventud del Ayuntamiento de Zaragoza en su página de Internet (www.cipaj.org/doment615n; accedido el 22/08/00).

Llegados a este punto, resulta oportuno realizar una breve recapitulación del estado de la investigación acerca del posible potencial adictivo de los videojuegos. Sólo mediante la visión global que esta revisión nos puede aportar estaremos en condiciones de formular opiniones sólidas, huyendo de prejuicios, alarmismos y primeras impresiones.

\section{¿QUÉ SE ENTIENDE POR "ADICCIÓN" EN LA INVESTIGACIÓN SOBRE LOS VIDEOJUEGOS?}

Actualmente estamos muy lejos de un consenso acerca de lo que entendemos por "adicción". Ni siquiera poseemos un lenguaje común para referirnos al proble- 
ma, siendo así que términos como "craving", "dependencia," "adicción", "trastorno adictivo" y "compulsión" son utilizados profusamente sin que exista acuerdo en relación al significado exacto de cada uno (Goodman, 1990). Y aún es menor el acuerdo acerca de la extensión del concepto, pues mientras algunos lo limitan únicamente al consumo de sustancias (p.ej., Jaffe, 1990), otros hablan de "adicciones no tóxicas" para referirse a diversas conductas problema, como el juego patológico (p.ej., Echeburúa, 1993) desordenes alimenticios (Lacey, 1993), "adicciones" sexuales (Myers, 1995), "adicción" al amor (Peele y Brodsky, 1975), "adicción" a correr por deporte (Morgan, 1979), "adicciones" tecnológicas genéricas (Griffiths, 1995), "adicción" a los ordenadores (Orzack, Friedman y Dessain, 1998), "adicción" a los videojuegos (Griffiths y Hunt, 1998; Soper y Miller, 1983) o "adicción" a Internet (Morahan-Martin, 1997), entre otras. Además, es importante tener en cuenta el uso coloquial del término tiende a confundir la adicción con el abuso e incluso con la afición, constituyendo con ello una fuente de confusión a la hora de interpretar los testimonios y las respuestas de los jugadores a las encuestas y cuestionarios.

Centrándonos en los estudios acerca de los videojuegos, lo primero que salta a la vista es que casi ninguno de los trabajos que han pretendido evaluar su potencial adictivo ha partido de una base teórica sólida acerca del fenómeno que se pretendía analizar. Ningún estudio ha hecho explícito el modelo de adicción del que se partía, y sólo en ocasiones se ha explicado el significado de los conceptos utilizados. Esto no carece de importancia, puesto que para admitir por ejemplo la presencia o la ausencia de tolerancia como prueba a favor o en contra de la adicción a los videojuegos, es preciso explicar por qué se adopta ese criterio, y no simplemente dar por sentado que tal hallazgo constituye una prueba. Esta carencia de base teórica hace que se hable de adicción basándose en aspectos como la frecuencia de juego, la duración de las jugadas, el dinero gastado en el juego o la comisión de actos reprobables para jugar. Con todo, aunque tales consideraciones son incluidas como síntomas de "dependencia" en el manual diagnóstico de la APA, no constituyen en sí mismas pruebas de adicción a menos que se asocien a una clara falta de control sobre la conducta de juego.

A continuación se revisan las características y resultados de las investigaciones que han abordado la cuestión de la adicción a los videojuegos. En la Tabla 1 se recoge un resumen de estas investigaciones.

\section{INVESTIGACIONES SOBRE LA ADICCIÓN A LOS VIDEOJUEGOS.}

Medidas de autoinforme y observacionales.
La mayoría de los datos disponibles acerca de la posible adicción a los videojuegos provienen de encuestas, mucho más asequibles que otros métodos de investigación pero de validez predictiva, sensibilidad y especificidad poco claras. Por otra parte, es muy reducido el número de estudios que han abordado directamente esta cuestión. Esto ha dificultado el diseño riguroso de los ítems relativos a la adicción, y con frecuencia ha desembocado en interpretaciones simplistas de los resultados.

A nivel general, los estudios basados en medidas de autoinforme coinciden en señalar la existencia de un grupo de sujetos cuyo uso de videojuegos es considerado "adictivo". Sin embargo, la interpretación que se da a estos resultados difiere con cada autor. Así, Brooks (1983) informaba de que tan sólo "una minoría" (no especificada) de sujetos se sentían empujados a jugar, de lo que concluía que el juego con videojuegos es una actividad mayoritariamente social, y no adictiva o compulsiva. También Greenfield (1984) señalaba que "aunque hemos encontrado algunos sujetos que se sentían empujados a jugar, se trataba de una minoría" (p.97; traducción nuestra) sin indicar datos referentes a porcentajes. Brown y Robertson (1990) encontraron resultados a favor de la existencia de adicción a los videojuegos en un "porcentaje notable" (no especificado) de la población. Sin duda, ante estos informes no cabe sino preguntarse qué se quiere decir con "porcentaje notable" o con "una minoría", y qué minoría es suficiente para causar preocupación.

Egli y Meyers (1984) también se basaban en un cuestionario para indicar que, aunque para la mayoría de jugadores el uso de videojuegos no constituye un problema, existe un porcentaje en torno al 10-15\% para quienes parece tratarse de una conducta "compulsiva". Se trataría de aquellos sujetos que juegan con mayor frecuencia, indican que se sienten adictos a los videojuegos, tienden a disfrutar con los videojuegos más que con cualquier otra actividad, y se muestran más inclinados a ser competitivos.

Utilizando un procedimiento diferente aunque también mediante un cuestionario, Rozin y Stoess (1993) evaluaron el potencial adictivo de los videojuegos en comparación con diversas sustancias y actividades potencialmente adictivas. Los videojuegos resultaron la actividad menos adictiva, únicamente por detrás de los juegos de azar.

Otros trabajos han abordado el problema de la adicción a los videojuegos centrándose en aspectos específicos del fenómeno adictivo. Así, McClure y Mears (1984) encontraban que el 15\% de los sujetos de su muestra jugaba a videojuegos para escapar de las presiones exteriores, si bien estos resultados fueron rebatidos por los mismos autores dos años más tarde (McClure y Mears, 1986). 
Tabla 1. Investigaciones sobre la adicción a los videojuegos.

\begin{tabular}{|c|c|c|c|c|}
\hline ESTUDIO & $N$ & EDAD & MÉTODO & RESULTADOS \\
\hline $\begin{array}{l}\text { Brooks } \\
(1983)\end{array}$ & 973 & & $\begin{array}{l}\text { Cuestionario a } \\
\text { jugadores }\end{array}$ & $\begin{array}{l}\text { Una minoría se sienten empujados a jugar; la mitad del tiempo en las salas se dedica a activi- } \\
\text { dades distintas del juego }\end{array}$ \\
\hline $\begin{array}{l}\text { Brown y } \\
\text { Robertson } \\
\text { (1990) }\end{array}$ & 45 & & $\begin{array}{l}\text { Items basados } \\
\text { en las } 20 \text { preguntas } \\
\text { para el juego a azar } \\
\text { compulsivo en } \\
\text { Jugadores Anónimos }\end{array}$ & Adicción a los VJs en un "porcentaje notable" (no especificado) \\
\hline $\begin{array}{l}\text { Creasey y } \\
\text { Myers (1986) }\end{array}$ & 47 & $9-16$ & $\begin{array}{l}\text { Cuestionario a usuarios } \\
\text { nuevos y antiguos de } \\
\text { VJs, en un intervalo } \\
\text { de } 4 \text { meses }\end{array}$ & $\begin{array}{l}\text { Importante decremento en juego en ambos grupos (jugadores nuevos: de } 15.4 \text { a } 6.3 \text { horas } \\
\text { semanales; jugadores antiguos: de } 8.1 \text { a } 2.3 \text { horas) }\end{array}$ \\
\hline $\begin{array}{l}\text { Eglli y } \\
\text { Meyers } \\
(1984)\end{array}$ & 151 & $10-20$ & $\begin{array}{l}\text { Cuestionario con } \\
\text { afirmaciones sobre } \\
\text { el carácter compulsivo } \\
\text { del uso de VJs }\end{array}$ & $\begin{array}{l}52 \% \text { juega más que un año atrás, } 18 \% \text { igual, } 30 \% \text { menos. Máximo de } 10-15 \% \text { de "adictos } \\
\text { a VJs". La mayoría no contempla el uso de VJs como un problema. Uso de VJs "compulsivo" } \\
\text { en los que juegan con más frecuencia, se sienten adictos, disfrutan más con los VJs que con } \\
\text { cualquier otra actividad, y son más competitivos }\end{array}$ \\
\hline $\begin{array}{l}\text { Estallo } \\
\text { (1993) }\end{array}$ & 65 & $12-18$ & $\begin{array}{l}\text { Registro del juego en } \\
\text { una ludoteca durante } \\
5 \text { meses }\end{array}$ & $\begin{array}{l}\text { Disminución lineal del tiempo que se dedica al VJ; reducción significativa de la duración media } \\
\text { de cada sesión de juego, en forma de curva de extinción }\end{array}$ \\
\hline $\begin{array}{l}\text { Fisher } \\
(1994 / 1995)\end{array}$ & 460 & $11-16$ & $\begin{array}{l}\text { Modificación de criterios } \\
\text { DSM-IV para juego } \\
\text { patológico }\end{array}$ & $\begin{array}{l}6 \% \text { adictos a los VJs. Adicción muy superior entre jugadores habituales (19\%) que entre } \\
\text { ocasionales (3\%) }\end{array}$ \\
\hline Griffiths (1998) & 5 & & Análisis de casos & $\begin{array}{l}\text { Los } 2 \text { que cumplen criterios de adicción tienen graves problemas en otros ámbitos. El uso } \\
\text { excesivo de VJs es sintomático, como defensa ante otras deficiencias }\end{array}$ \\
\hline $\begin{array}{l}\text { Griffiths y } \\
\text { Dancaster } \\
\text { (1995) }\end{array}$ & 60 & 24 & $\begin{array}{l}\text { Modificación de criterios } \\
\text { DSM-III-R para juego } \\
\text { patológico }\end{array}$ & $37.5 \%$ son adictos a los VJs o lo han sido en algún momento de su vida \\
\hline $\begin{array}{l}\text { Griffiths y } \\
\text { Hunt (1998) }\end{array}$ & 387 & & $\begin{array}{l}\text { Modificación de criterios } \\
\text { DSM-III-R para juego } \\
\text { patológico }\end{array}$ & $\begin{array}{l}19.9 \% \text { de adictos a los VJs; } 6.8 \% \text { suele jugar a niveles de adicción. Correlación positiva de las } \\
\text { puntuaciones en adicción con sexo (más en chicos), frecuencia de juego, y duración media y } \\
\text { máxima por sesión; negativa con edad de inicio. } 33 \% \text { considera que los VJs son adictivos }\end{array}$ \\
\hline Keepers (1990) & 1 & 12 & Análisis de casos & Juego como respuesta parcialmente adaptativa \\
\hline Klein (1984) & & & Análisis de casos & $\begin{array}{l}\text { Preocupación por los VJs en niños con trastornos de oposición o de conducta. Padres y profe- } \\
\text { sores informan que los niños gastan dinero del almuerzo en VJs, y de que roban o piden para } \\
\text { jugar }\end{array}$ \\
\hline $\begin{array}{l}\text { McClure y } \\
\text { Mears (1984) }\end{array}$ & 336 & $9-10$ & $\begin{array}{l}\text { Cuestionario a } \\
\text { estudiantes }\end{array}$ & $15 \%$ juega a VJs para escapar de las presiones exteriores \\
\hline $\begin{array}{l}\text { McClure y } \\
\text { Mears (1986) }\end{array}$ & 290 & $14-17$ & $\begin{array}{l}\text { Cuestionario a } \\
\text { estudiantes; } \\
\text { EPI, MMPI, CPI }\end{array}$ & $\begin{array}{l}\text { Jugadores habituales no informan de menor felicidad familiar, no tienen más probabilidad de } \\
\text { pertenecer a familia monoparental o con un padre no biológico, ni de fugarse de sus } \\
\text { domicilios }\end{array}$ \\
\hline $\begin{array}{l}\text { Phillips, Rolls, } \\
\text { Rouse y } \\
\text { Griffiths (1995) }\end{array}$ & 868 & $11-16$ & $\begin{array}{l}\text { Cuestionario a estud.; } \\
\text { modificación de criterios } \\
\text { DSM-III-R para juego } \\
\text { patológico }\end{array}$ & $\begin{array}{l}7.5 \% \text { de adictos a los VJs; estos juegan } 6 \text { ó más días a la semana, }>1 \text { h. por sesión, más tiem- } \\
\text { po del pensado, y no hacen las tareas escolares para jugar. Porcentajes importantes cumplen } \\
\text { al menos algún criterio: jugar más tiempo del pretendido }(60.6 \%)\end{array}$ \\
\hline $\begin{array}{l}\text { Rozin y } \\
\text { Stoess (1993) }\end{array}$ & 573 & & $\begin{array}{l}\text { Valoración de síntomas } \\
\text { de adicción a VJs y otras } \\
\text { sustancias y conductas }\end{array}$ & $\begin{array}{l}\text { VJs son la actividad menos adictiva tras juegos de azar. No correlación entre puntuación de los } \\
\text { jóvenes y puntuación media de sus padres, pero sí entre puntuación de padre y madre. Corre- } \\
\text { lación negativa entre puntuación de adicción a VJs y consumo de café, y positiva con consu- } \\
\text { mo de bebidas de cola, de alcohol, y con juegos de azar }\end{array}$ \\
\hline Tejeiro (1998) & 1358 & 14 & $\begin{array}{l}\text { Modificación de criterios } \\
\text { DSM-IV para juego } \\
\text { patológico }\end{array}$ & $\begin{array}{l}\text { Dependencia (14.9\%), preocupación mientras no se está jugando (11.4\%), uso como meca- } \\
\text { nismo de evasión }(8.2 \%) \text {, abstinencia }(5.7 \%) \text {, tolerancia }(4.7 \%) \text {. Niños superan a niñas en } \\
\text { todos los síntomas. Relación positiva entre adhesión al juego y (a) presencia de cada síntoma, } \\
\text { y (b) número de síntomas de cada sujeto }\end{array}$ \\
\hline Turkle (1984) & & & Análisis de casos & Rechaza la noción de "adicción" a los juegos de ordenador \\
\hline
\end{tabular}


Respecto a la posibilidad de tolerancia a los videojuegos, Creasey y Myers (1986) y Estallo (1993) demostraron que la dedicación a los videojuegos muestra un decremento con el tiempo a partir del momento en que se introduce un nuevo juego o una nueva máquina de juegos en la vida del joven. Estos autores consideran este hallazgo como muestra de la ausencia de tolerancia a los videojuegos, llegando a proponerlo como prueba de la inexistencia de adicción a los mismos. Con todo, en el análisis de estos estudios hay que tener presente que el DSM-IV (APA, 1994/1995) indica que "ni la tolerancia ni la abstinencia son condiciones necesarias ni suficientes para diagnosticar una dependencia" (p.184), aunque, de hecho, el manual reserve la denominación de "dependencia" únicamente para la adicción a sustancias tóxicas.

Por otra parte, todos los estudios acerca del potencial de los videojuegos para generar adicción se han enfrentado a una importante limitación: la falta de un sistema diagnóstico válido y fiable. Algunos autores (p.ej., Shotton, 1989) se han limitado a obviar esta limitación, aceptando que los sujetos analizados eran "dependientes de los juegos de ordenador", simplemente porque se calificaban a sí mismos como tal. Otros trabajos han intentado solventar esta carencia incorporando sistemas diagnósticos desarrollados a partir de criterios propuestos para la identificación y medida del juego patológico. Aunque el manual de la APA clasifica el juego patológico dentro de los "trastornos del control de los impulsos", son numerosos los que lo consideran como una adicción no tóxica (ver Echeburúa, 1993). Por consiguiente, su adaptación para el diagnóstico de otra posible adicción no tóxica se ha presentado como un paso adelante en la objetivación de las investigaciones.

Sin embargo, aunque está muy extendida la creencia en la relación entre los videojuegos y los juegos de azar, hasta la fecha no se ha demostrado el paralelismo entre la posible adicción a unos y a otros. Los estudios que argumentan a favor de tal semejanza provienen fundamentalmente del Reino Unido, y se basan en las características que comparten las máquinas recreativas con y sin premio económico (p.ej., Blackpool and Fylde Youth Community Service, 1991; Huxley y Carroll, 1992). No obstante, en la interpretación de los resultados es preciso tener en cuenta, en primer lugar, que todos estos estudios se centran exclusivamente en los videojuegos que utilizan como soporte máquinas operadas con monedas. Además, en el Reino Unido las máquinas de videojuegos comparten salones con las máquinas tragaperras, ya que se trata del único país occidental en el que se permite a los niños jugar con estas últimas (Fisher, 1995), y el control sobre la conducta de juego con tragaperras en los menores depende de un código voluntario de escaso cumplimiento, establecido y administrado por los operadores de las salas (Griffiths, 1991a). Estos hechos han llevado a algunos autores a confundir ambos tipos de máquinas a la hora de analizar los datos de las investigaciones, y no es infrecuente encontrar comentarios referentes a las "máquinas recreativas", sin especificar a qué tipo de máquinas se hace alusión.

La primera propuesta de adaptación de los criterios de juego patológico del DSM-III-R (APA, 1987) para medir la de adicción a los videojuegos la realizó Griffiths (1991b). La adaptación propuesta por este autor incluía 9 criterios, de los cuales se tienen que cumplir al menos 4 para considerar al sujeto como un probable adicto, aunque no se aportaban datos sobre las propiedades psicométricas de la escala. La adaptación de los criterios de juego patológico del DSM-IV (APA, 1994) la realizó Fisher (1994, 1995), quien desarrolló la escala DSM-IV-JV ( $\mathrm{J}=$ juvenil; $\mathrm{V}=$ videojuegos de arcade). Esta escala se compone de 9 ítems, de los cuales deben cumplirse al menos 4 para la emisión del diagnóstico. En este caso sí se informaban las propiedades psicométricas, con valores aceptables. Con todo, hay que señalar que la escala DSM-IV-JV se centra exclusivamente en los videojuegos de salas recreativas, introduciendo así un sesgo al otorgar una notable importancia al componente económico, que resulta relevante únicamente en el caso de las máquinas de videojuegos operadas con monedas. Esto limita notablemente la aplicación de la escala, ya que el porcentaje de uso de dicho sistema es muy inferior al de los sistemas domésticos (Tejeiro, 1998).

Los trabajos que han utilizado adaptaciones de los criterios del manual de la APA encuentran resultados diversos, desde el $6 \%$ de jugadores adictos (Fisher, 1994, 1995) hasta el 37'5\% que, según Griffiths y Dancaster (1995), eran adictos a los videojuegos o lo habían sido en algún momento de su vida, pasando por el 7' $5 \%$ de adictos identificados por Phillips, Rolls, Rouse y Griffiths (1995). Griffiths y Hunt (1998), por su parte, identificaron un 19 '9\% de jugadores adictos a los juegos de ordenador, con un 6' $8 \%$ adicional que solía jugar a niveles de adicción. Con todo, en sus conclusiones los autores señalan que la falta de asociación entre las consecuencias negativas del juego con videojuegos (robo, absentismo escolar, etc.) y las puntuaciones en dependencia parece respaldar la posibilidad de que la escala utilizada sea más una escala de preocupación que de dependencia.

En nuestro país, hace unos años utilizamos también cinco preguntas adaptadas a partir de los criterios del DSM-IV para el juego patológico, en una investigación general acerca de las variables asociadas al uso de videojuegos (Tejeiro, 1998). Pudimos así constatar una importante presencia de diversos síntomas de adicción, aunque la falta de análisis de las propiedades psicométricas de la escala impidió el establecimiento de puntos de corte y la valoración del nivel de "adicción" de los sujetos de la muestra. 
Análisis de casos.

Algunos trabajos se han basado en el análisis de casos para estudiar la posible adicción a los videojuegos. Esta aproximación carece del control estadístico de las investigaciones basadas en encuestas, pero posibilita una relación mucho más estrecha entre el investigador y el sujeto $y$, por consiguiente, permite un estudio pormenorizado de aspectos que, de otra manera, pasarían desapercibidos.

Algunos estudios que han adoptado esta perspectiva se limitan a constatar la relación entre el uso de videojuegos y síntomas de adicción (Klein, 1984; Turkle, 1984). Otros trabajos profundizan en los orígenes de los síntomas que muestran los sujetos. Así, Keepers (1990) presentaba el caso de un niño cuya dedicación excesiva a los videojuegos le había acarreado serios problemas. Durante el tratamiento, se pudo comprobar que el juego era, de hecho, una respuesta parcialmente adaptativa: el tiempo que el niño pasaba en las salas recreativas le ayudaba a mantenerse físicamente fuera de una tensa situación doméstica. De manera semejante, Griffiths (1998) analizaba cinco casos de uso excesivo de los juegos de ordenador, encontrando que aquellos podían cumplir los criterios de "adicción" presentaban también graves problemas en otros ámbitos (problemas de relación, desagrado con su aspecto físico, abuso o maltrato por parte de los padres, etc.). Este autor señalaba que el uso excesivo de los videojuegos en estos casos era meramente sintomático, y que el ordenador era utilizado como defensa ante las otras deficiencias. Otros autores (Muñóz, 1999) avalan estos planteamientos a partir de su propio trabajo clínico.

\section{Investigación sobre neurotransmisores.}

Un equipo de investigación dirigido por el Dr. Paul Grasby, del Hammersmith Hospital de Londres, llevó a cabo en 1998 un estudio destinado a analizar la producción de dopamina en el cerebro humano durante una experiencia de aprendizaje orientada a metas, utilizando en el experimento un videojuego (Vanous, 1998). Encontraron así que los niveles de dopamina se incrementaban de forma notable durante el juego, hasta casi el doble de su valor inicial, aumento que es comparable al que produce el consumo de las anfetaminas. Aunque los hallazgos son aún prematuros y admiten numerosas interpretaciones alternativas, se sugiere que pueden suponer la primera prueba científica de que los videojuegos generan adicción física.

\section{CONSIDERACIONES FINALES}

La mayoría de los trabajos revisados tienden a señalar que, para algunos sujetos, el uso de videojue- gos constituye un problema. Sin embargo, permanece acierta la cuestión sobre la naturaleza y el origen de dicho problema. Al respecto, hay algunas propuestas que no han pasado de la mera especulación teórica. Por ejemplo, Griffiths (1993) propone cuatro posibles mecanismos explicativos de la adicción a los videojuegos: (1) como función de los efectos del juego sobre la imaginación y la fantasía; las personas que usan los videojuegos en exceso tienen poca imaginación; (2) como función de los efectos del videojuego sobre el nivel de arousal; los que usan los videojuegos en exceso lo hacen bien por sus efectos activadores, bien por sus efectos tranquilizantes; (3) como manifestación de la personalidad oral, dependiente o adictiva; y (4) como patrón distintivo de usos y gratificaciones asociado con el medio en que se usa el videojuego; las personas que juegan en exceso disfrutan del acto físico de jugar, o juegan sólo cuando se encuentran aburridos.

Cada una de estas posibilidades sugiere interesantes vías de estudio y está sujeta a críticas que exceden el objeto de la presente revisión. Por otra parte, cabe preguntarse si no estaremos aplicando la etiqueta de "adicción" a cualquier conducta aparentemente excesiva. ¿Son adictos los jugadores que utilizan los videojuegos "sólo cuando se encuentran aburridos", por más que pasen horas y horas ante el ordenador o la consola? Probablemente en ese caso, como en otros, sea más apropiado hablar de "abuso" que de "adicción", e incluso para emitir tal diagnóstico hay que tener en cuenta otras variables que, aún siendo importantes de cara a un análisis global del problema, suelen ser alegremente omitidas por los estudios: el tipo de videojuego que utiliza la persona (Griffiths y Hunt, 1998), el papel que desempeñan los videojuegos dentro del mundo de relaciones del joven (Herz, 1997; Suess, Suoninen, Garitaonandía, Juaristi, Koikkalainen y Oleaga, 1998), su papel dentro de su propio proceso madurativo del usuario (Turkle, 1994), etc.

Por otra parte, es posible que, en el caso de que exista la adicción a los videojuegos, esta tenga características diferentes para grupos diferentes de personas. En este sentido propone Griffiths (1993) que pueden existir dos tipos de adicciones a las máquinas de videojuegos. Los adictos del primer tipo, que denomina "adicción primaria", serían adictos a la máquina en sí, y jugarían por el incremento en el arousal que produce la excitación del juego, así como por obtener recompensas sociales y para poner a prueba sus habilidades. El otro tipo ("adicción secundaria") recurriría al juego con videojuegos como actividad de desplazamiento o para reducir el estrés que le producen otras áreas de la vida. Este segundo tipo trata a los videojuegos como "amigos electrónicos" (Selnow, 1984), y se ajusta al estereotipo de "adicto" a los videojuegos aislado socialmente. 
Algunos autores han revisado sus trabajos a la luz de esta propuesta, concluyendo que los sujetos que han identificado como posibles adictos a los videojuegos pueden ser encuadrados en el grupo de "adicción secundaria" (Phillips, Rolls, Rouse y Griffiths, 1995). También los resultados de los análisis de casos revisados parecen apuntar a la presencia de dicha "adicción secundaria".

En definitiva, en nuestra revisión hemos podido constatar que a pesar de que la posible existencia de adicción a los videojuegos constituye un problema que levanta considerable preocupación social, existe una llamativa carencia de estudios centrados en este tema. Por otra parte, los estudios tienden a apoyarse en un frágil soporte teórico, adolecen de una visión global de la cuestión, muestran notables diferencias metodológicas, y las replicaciones son escasas, por lo que los resultados son parciales y sujetos a múltiples interpretaciones. Dada la creciente importancia de los videojuegos en la cultura de los niños y adolescentes, es preciso solventar todas estas carencias en la investigación, diseñando y realizando trabajos específicos, metodológicamente apropiados, con suficiente respaldo teórico, y con una visión global del papel de los videojuegos en el mundo del usuario. Mientras tanto, cualquier posicionamiento a favor o en contra del posible carácter adictivo de los videojuegos constituye más una declaración de principios que un reflejo objetivo de los hallazgos.

\section{BIBLIOGRAFÍA}

American Psychiatric Association (1987). Diagnostic and statistical manual of mental disorders, (3 ${ }^{\text {rd }}$. ed, Rev.). Washington, DC: Author.

American Psychiatric Association (1994). Diagnostic and statistical manual of mental disorders, (4th ed.). Washington, DC: Author (trad. cast. en Barcelona: Masson, 1995).

Blackpool and Fylde Youth Community Service (1991). A research study on young people and gambling in Blackpool. Blackpool: Author.

Brown, R.I.F. y Robertson, S. (1993). Home computer and video game addictions in relation to adolescent gambling: Conceptual and developmental aspects. En W.R. Eadington and J.A. Cornelius (Eds.), Gambling behavior and problem gambling. Reno, NV: University of Nevada in Reno.

Creasey, G.L.. y Myers, B.J. (1986). Video Games and children: Effects on Leisure Activities, Schoolwork and Peer Involvement. Merrill-Palmer Quarterly, 32 (3), 251262.

Echeburúa, E. (1993). Las conductas adictivas: ¿Una ruta común desde el crack al juego patológico? Psicología Conductual, 1 (3), 321-337.
Egli, E.A. y Meyers, L.S. (1984). The role of videogame playing in adolescent life: Is there reason to be concerned?. Bulletin of Psychonomic Society, 22 (4), 309-312.

Estallo, J.A. (1993). Videojuegos. Efectos sobre el comportamiento. Texto no publicado. Institut Psiquiàtric. Barcelona.

Fisher, S.E. (1994). Identifying video game addiction in children and adolescents. Addictive Behaviors, 19 (5), 545-553.

Fisher, S.E. (1995). The amusement arcade as a social space for adolescents: an empirical study. Journal of Adolescence, 18, 71-86.

Goodman, A. (1990). Addiction: definition and implications. British Journal of Addiction, 85, 1403-1408.

Greenfield, P.M. (1984). Mind and Media: The Effects of Television, Video Games and Computers. Cambridge, MA: Harvard University Press.

Griffiths, M.D. (1991a). The observational study of adolescent gambling in U.K. amusement arcades. Journal of Community and Applied Social Psychology, 4 (1), 309-320.

Griffiths, M.D. (1991b). Amusement machine playing in childhood and adolescence: A comparative analysis of video games and fruit machines. Journal of Adolescence, 14 (1), 53-73.

Griffiths, M.D. (1993). Are computer games bad for children?. The Psychologist: Bulletin of the British Psychological Society, 6 (9), 401-406.

Griffiths, M.D. (1995). Technological addictions. Clinical Psychology Forum, 76, 14-19.

Griffiths, M.D. (1998). Does Internet and Computer 'Addiction' Exist?: Some Case Study Evidence. Paper presented at the IRISS'98 Internet Research and Information for Social Scientists, Bristol, UK, March.

Griffiths, M.D. y Dancaster, I. (1995). The effect of Type A personality on physiological arousal while playing computer games. Addictive Behaviors, 20 (4), 543-548.

Griffiths, M.D. y Hunt, N. (1998). Dependence on Computer Games by Adolescents. Psychological Reports, 82, 475-480.

Herz, J.C. (1997). Joystick Nation. London: Abacus.

Huxley, J. y Carroll, D. (1992). A survey of fruit machine gambling in adolescents. Journal of Gambling Studies, 8 (2), 167-179.

Jaffe, J.H. (1990). Trivializing dependence. British Journal of Addiction, 85, 1425-1427.

Keepers, G.A. (1990) Pathological Preoccupation with Video Games. Journal of the American Academy of Child and Adolescent Psychiatry, 29 (1), 49-50.

Klein, M.H. (1984). The bite of Pac-man. Journal of Psychohistory, 11, 395-401.

Lacey, H.J. (1993). Self-damaging and addictive behaviour in bulimia nervosa: a catchment area study. British Journal of Psychiatry, 163, 190-194.

McClure, R.F. y Mears, F.G. (1994). Video game players: personality characteristics and demographic variables. Psychological Reports, 55, 271-276. 
McClure, R.F. y Mears, F.G. (1986). Videogame Playing and Psychopatology. Psychological Reports, 59, 59-62.

Morahan-Martin, J. (1997, August). Incidence and correlates of pathological Internet use. Paper presented at the $105^{\text {th }}$ annual meeting of the American Psychological Association, Chicago, IL.

Morgan, W.P. (1979). Negative addiction in runners. Physician and Sportsmedicine, 7 (2), 55-70.

Muñoz, S. (1999). ¿Son dañinos los videojuegos, la televisión y la computadora?. Accedido el 28 de Junio de 2000 en la World Wide Web: http://www.mipediatra.com.mx/ videojue.htm

Myers, W.A. (1995). Addictive sexual behavior. American Journal of Psychotherapy, 49 (4), 473-483.

Orzack, M.H., Friedman, L. y Dessain, E. (1998). Comparative study of the abuse liability of alprazolam, lorazepam, diazepam, methaqualone, and placebo. International Journal of the Addictions, 23 (5), 449-467.

Peele, S. y Brodsky, A. (1975). Love and Addiction. New York: Taplinger.

Phillips, C.A., Rolls, S., Rouse, A., y Griffiths, M.D. (1995). Home video game playing in schoolchildren: a study of incidence and patterns of play. Journal of Adolescence, $18(6), 687-691$.
Rozin, P. y Stoess, C. (1993). Is there a general tendency to become addicted? Addictive Behaviors, 18 (1), 81-87.

Selnow, G.W. (1984). Playing video games. The electronic friend. Journal of Communication, 34 (2), 148-156.

Shotton, M. (1989). Computer addiction? A study of computer dependency. London: Taylor and Francis.

Soper, W.B. y Miller, M.J. (1983). An emerging addiction among students. School Counselor, 31 (1), 40-43.

Suess, D., Suoninen, A., Garitaonandía, C., Juaristi, P., Kokkalainen, R. y Oleaga, J.A. (1998). Media use and the relationships of children and teenagers with their peer groups. A study of Finnish, Spanish and Swiss cases. European Journal of Communication, 13 (4), 521-538.

Tejeiro, R. (1998). La práctica de videojuegos en niños del Campo de Gibraltar. Algeciras: Asociación de Jugadores de Azar en Rehabilitación del Campo de Gibraltar.

Turkle, S. (1984). The Second Self: Computers and the Human Spirit. London: Granada.

Vanous, C. (1998). Games: An Addiction? Game News, October 12. Accedido el 15 de Julio de 2000 en la World Wide Web: http://204.162.80.132/News/Item/ 0,3,0-2172,00.html 
